\title{
Design and Dynamic Analysis of a Four-Degree-of-Freedom Chaotic Vibrating Screen
}

\author{
Mingkun Zhang $\mathbb{D}^{1,2}$ Chengjun Wang, ${ }^{1,2}$ Chen Yan, ${ }^{1}$ and $\mathrm{Hao} \mathrm{Li}^{1}$ \\ ${ }^{1}$ Mechanical Engineering Institute, Anhui University of Science and Technology, Huainan City 232001, China \\ ${ }^{2}$ Anhui Key Laboratory of Mine Intelligent Equipment and Technology, Anhui University of Science and Technology, \\ Huainan City 232001, China
}

Correspondence should be addressed to Mingkun Zhang; hwjin@aust.edu.cn

Received 21 July 2020; Revised 30 March 2021; Accepted 19 April 2021; Published 7 May 2021

Academic Editor: Gianluca Gatti

Copyright (c) 2021 Mingkun Zhang et al. This is an open access article distributed under the Creative Commons Attribution License, which permits unrestricted use, distribution, and reproduction in any medium, provided the original work is properly cited.

\begin{abstract}
In order to address the problems of low screening efficiency, easy blocking of screen holes, and short service life of key parts commonly used in vibrating screen equipment, the TRIZ (Theory of the Solution of Inventive Problems) was applied in the present work to design a four-degree-of-freedom (4-DOF; three translational and one rotational movements) chaotic vibrating screen with a chaotic vibration exciter as the main power source and a 3-DOF (three translational movements) parallel kinetic chain as the kinematic constraint mechanism of the outer screen frame. Based on the topological structure theory, a hybrid mechanism with structure [4SOC $\left.\left\{-C_{i 1}\left\|R_{i 2}\right\| R_{i 3}-\right\}+R,(i=1,2,3,4)\right]$ was constructed as the kinematic constraint mechanism of the inner screen box of the chaotic vibrating screen to solve the freedom of motion and POC (position and orientation characteristic) equations of parallel kinematic constraint mechanism of the outer screen frame and hybrid constraint mechanism of the inner screen box. The dynamic simulation of a virtual prototype of the chaotic vibrating screen was carried out in ADAMS software, and MATLAB was used to chaos recognition of the simulation results. It was found that the chaotic exciter moved aperiodically in $X$-, $Y$-, and $Z$-directions when the chaotic exciter motor rotated at uniform speed, and the amplitude, velocity, and acceleration of the outer screen frame of the vibrating screen had characteristics of reciprocating aperiodic and irregular changes. Through the phase trajectories of the eccentric block and inner screen box of the exciter in all directions, it was observed that the motion output of the vibrating screen was a chaotic vibration. Therefore, the present paper can provide an important reference for the design and application of chaotic vibrating screens.
\end{abstract}

\section{Introduction}

In 1960 [1], Lorenz first proposed the butterfly effect and expounded the basic features of chaos. In 1963, Lorenz [2] published a paper entitled "Decisive aperiodic flow" and performed a preliminary study on chaos by numerical simulations. Krysko et al. [3] studied the chaotic dynamics of flexible spherical axially symmetric shallow shells subjected to a sinusoidal transverse load, solved the governing partial nonlinear equations, estimated the signs of the largest Lyapunov exponents, and verified the reliability of chaos. Kim et al. [4] investigated the chaotic vibration of a rotor system with an asymmetric nonlinear stiffness and derived the motion equation of the rotor in a fixed coordinate system under Shilnikov chaotic vibration. Liu et al. [5] adopted the theory of double-potential well to design a single-ended magnetic suction-type chaos vibration test device and studied its responses under different excitation amplitudes and frequencies. It was found that the system could produce sustainable and stable vibration under certain frequencies and excitation amplitudes.

Malanchuk et al. [6] from the National Academy of Sciences of Ukraine conducted a study on prescreening of basalt raw materials in order to extract iron, titanium, and copper elements from basalt. Makinde et al. [7, 8] designed a reconfigurable vibrating screen with an adjustable screen structure and a multifunctional robotic end-effector that can perform all maintenance tasks on the device and studied the 
maintenance technology and maintenance standards of the device when working in the mine environment, so as to meet the requirements of customers for sorting and screening mineral particles of different sizes and quantities. Li [9] designed a three-degree-of-freedom (3-DOF) chaotic vibrating screen through numerical simulations based on the chaotic phenomenon of the linkage mechanism with kinematic pair clearance. Qin et al. [10] designed an underactuated chaotic vibrating screen with a redundant 1 -DOF. The Lagrange method was used to develop the dynamic model of the mechanism, and the chaotic motion of the screen box was verified through the numerical simulation method. Chen et al. [11] studied the influence of rotational speeds of key components on the chaotic motion of a chaotic vibrating screen. Wang et al. [12] established a mathematical model for a mining chaotic vibrating screen and analyzed all of the necessary conditions of chaotic vibration. Wang and Long [13] from China Agricultural University showed that chaotic vibration has wider vibration frequency and greater acceleration variation than periodic vibration and can be used for vibration compaction, vibration screening, vibration cutting, vibration blanking, vibration aging, and broadband vibration test. Wang et al. [14] designed a 3-DOF chaotic vibrating screen using both parallel and hybrid mechanisms. Song et al. [15] designed a new type of multiDOF chaotic vibrating screen based on the principle of multi-DOF dynamics. A high screening efficiency was obtained by eliminating the blind hole and adding high-frequency, short-amplitude vibration.

A chaotic vibrating screen is a kind of vibrating screen that makes the screen box produce chaotic vibration through the nonlinear vibration of the exciter. TRIZ (Theory of the Solution of Inventive Problems) theory is an efficient invention problem-solving theory founded by Althshuller [16], an inventor of the former Soviet Union, in 1946. It is a mature technical method suitable for solving practical problems in product development, with high innovation efficiency, wide application range, and strong operability. In the present work, a 4-DOF (three translational and one rotational movements) chaotic vibrating screen was designed based on the TRIZ theory. The feature of this design is that the vibrating screen used a chaotic vibration exciter as the main power source and a parallel kinematic chain for the kinematic constraint mechanism of the outer screen frame, so that the outer screen frame produces chaotic vibration, which can improve the load capacity and screening efficiency of the vibrating screen and overcome the deficiency of the existing vibrating screen. Based on the topology theory of mechanism, the topological structure of the chaotic vibrating screen was created and its freedom of motion was calculated. The dynamic simulation of the vibrating screen was performed in ADAMS software, and MATLAB was used for chaos recognition.

At present, linear and circular vibrating screens are mostly used in industries; they pose some common problems, such as low screening efficiency, easy blocking of screen holes, and short service life of key components. Thus, it is difficult to separate viscous and moist materials and screen mass materials $[14,17,18]$. Chaotic vibration has a wider vibration frequency than linear vibration, and it causes more severe velocity change, which in turn strengthens the flow state between material particles. The application of chaotic vibration in the design of vibrating screens can increase the contact area between the material particles and screen surface and enable the screening of viscous and moist materials. The screening efficiency under chaotic vibration is much higher than that under simple harmonic vibration $[15,19,20]$.

\section{Structure of the 4-DOF Chaotic Vibrating Screen}

Existing vibrating screens mostly work under linear vibration with less degrees of freedom and experience several problems, such as low screening efficiency, poor screening effect, and short service life of key components. Thus, it is difficult to separate viscous and moist materials and screen materials with high viscosity. Therefore, it is necessary to design a new type of multi-DOF vibrating screen that can provide nonlinear motion, improved screening effect, and sufficient blanking. In the present work, a 4-DOF (three translational and one rotational movements) chaotic vibrating screen was developed based on the TRIZ theory.

2.1. Nine-Screen Method and Goldfish Method. The ninescreen method was used to design a multi-DOF vibrating screen to overcome the existing problems in the blanking of vibrating screens, and the analysis is depicted in Figure 1.

The goldfish method is a method to overcome the inertia of thinking in TRIZ theory. It transforms the fantasy and unrealistic problem solution into a feasible solution by constantly distinguishing the real and fantasy parts from the problem-solution concept. To meet the multi-DOF nonlinear vibration screening requirements of viscous and moist materials, the goldfish method was adopted to design a 4DOF vibrating screen with chaotic vibration characteristics.

2.2. Technical Contradiction Analysis. In the TRIZ conflict matrix shown in Table 1, structural stability (No. 13), adaptability, and versatility (No. 35) are the deterioration parameters, and equipment complexity (No. 36) is the improved parameter.

Combined with the actual problems existing in the vibrating screen, through the analysis, the following can be found: invention principles 17 (spacial dimension change), 19 (periodic action), and 15 (dynamic) had reference value for solving the technical contradiction. The solution was as follows:

(1) Invention Principle 17 (Principle of Spatial Dimension Change). The screen box was divided into two parts-the outer screen frame and the inner screen box with a relative motion. The outer screen frame drove the inner screen box to realize three translational motions. According to principle 17 (objects arranged in a single layer become arranged in multilayers), all four of the symmetrically arranged 


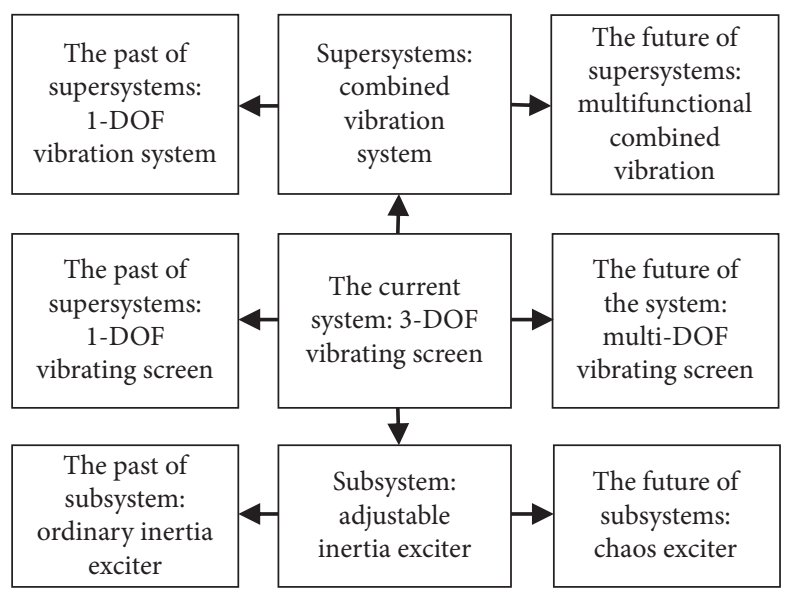

FIGURE 1: Nine-screen analysis of the vibrating screen.

TABLe 1: Conflict matrix.

\begin{tabular}{lccc}
\hline Deteriorating parameters improved parameter & 13 & 35 \\
& Equipment complexity & Structural stability & Adaptability and versatility \\
\hline 36 & $2,22,17,19$ & $29,15,28,37$ \\
\hline
\end{tabular}

constraint chains in both vertical and horizontal directions were used for the kinematic constraint mechanism of the vibrating screen to increase the structural strength and stability. The design scheme is displayed in Figure 2.

(2) Principle 19 (Principle of Periodic Action). The new exciter changed its motion frequency according to the periodic excitation of the original exciter or used the pulse between the two propelling forces to provide an extra force. According to the design of the chaotic exciter, complex chaotic vibration was generated under simple excitation by enlarging the nonlinear factor of the vibrating screen. The structure of the chaotic exciter for the vibrating screen after the innovative design is presented in Figure 3.

The chaotic exciter consisted of a rotary motor, an eccentric block, a braced seat, and a shell. The left and right sides and bottom of the braced seat were connected with the shell of the exciter through linking springs. The eccentric block was installed at both ends of the rotary motor, which acted as the excitation force source of the chaotic exciter.

(3) Principle 15 (Principle of Dynamics). The rotational degree of freedom of the vibrating screen was used based on principle 15 (making a static object moving or movable). The inner screen box and outer screen frame of the vibrating screen were connected with a coaxial revolving support device to realize the back-and-forth rotation of the inner screen box around the transverse horizontal axis. The excitation was realized through the electromagnetic exciter installed on one side of the screen. As the outer screen frame needed to achieve three translational motions, it was connected with a bracket through a suspension spring with spherical hinges at both ends (Figure 4).

2.3. Determination of Design Scheme. The final design of the 4-DOF (three translational and one rotational movements) chaotic vibrating screen is presented in Figure 5. The outer screen frame was hoisted under the bracket by four sets of suspension springs. The outer screen frame and the support were connected by four pairs of constraint chains of a CRR structure. The longitudinal constraint chain was symmetrically arranged on the left and right sides of the outer screen frame, and the upper end of the outer screen frame was arranged horizontally to the constraint chain to form a parallel constraint mechanism of a $2 \mathrm{CRR}$ structure, thus realizing three translational motions of the outer screen frame. Using the nonlinear characteristics of the suspension spring, the vibrating screen absorbed a part of the excitation energy of the chaotic exciter and caused system vibration isolation to produce more ideal vibration output by energy superposition.

The base plate of the chaotic exciter was fixedly connected with the outer screen frame, the eccentric block delivered energy to the base plate by connecting spring, and then the outer screen frame drove the inner screen box to realize reciprocating vibration in the $X-, Y$-, and $Z$ - directions. Driven by the electromagnetic exciter, the inner screen box generated reciprocating rotating vibration along the $X$ axis relative to the outer screen frame through the revolving support device. Therefore, the inner screen box had three translational and one rotational movements, a total of 4DOF movement. Because the kinematic constraint 


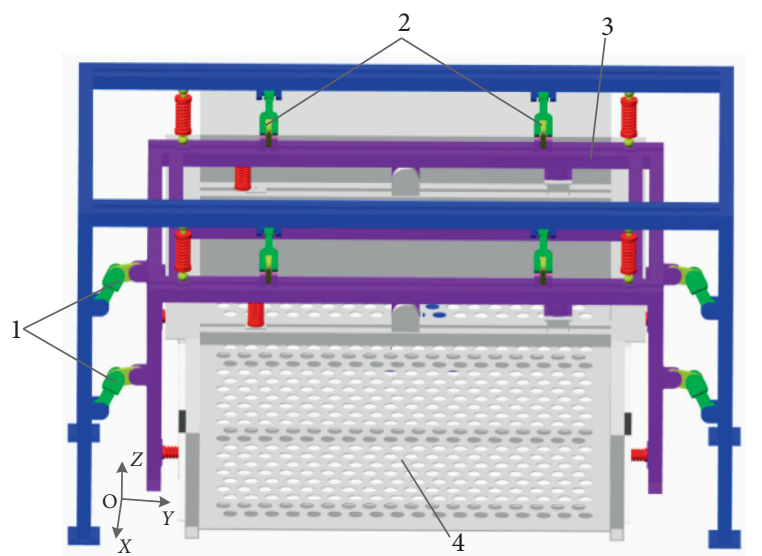

FIGURE 2: Spatial dimension change principle. 1: vertical constraint chain; 2: horizontal constraint chain; 3: outer screen frame; 4 : inner screen box.

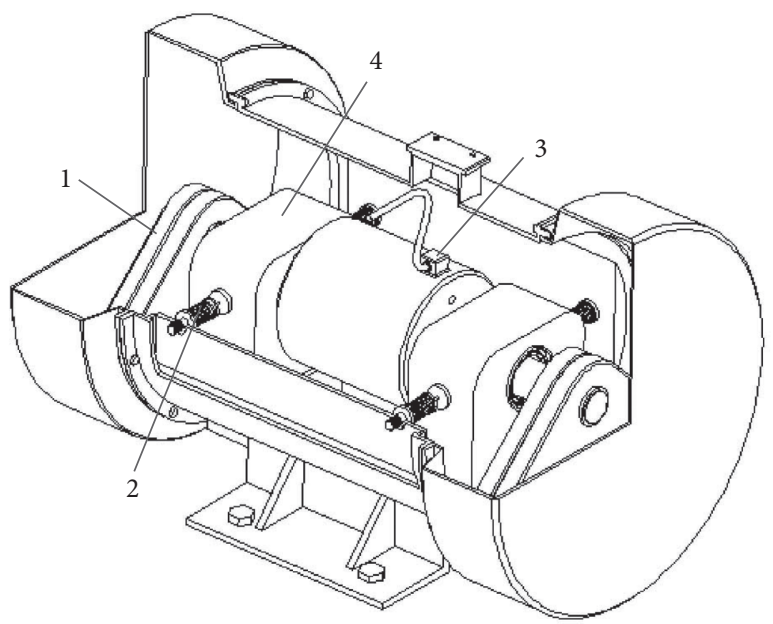

Figure 3: Chaotic exciter. 1: eccentric block; 2: connecting spring; 3: rotary motor; 4 : braced seat.

mechanisms of the outer screen frame and the inner screen box were 3-DOF parallel mechanism and 4-DOF hybrid mechanism, respectively, it had the advantages of high structural stiffness and accurate motion, which could guarantee the kinematic accuracy and reliability of the screen surface of the vibrating screen at work.

\section{Analysis of Kinematic Constraint Mechanism of the Chaotic Vibrating Screen}

3.1. Topological Analysis of Parallel Kinematic Constraint Mechanism. The kinematic constraint mechanism of the 4DOF chaos vibration screen was designed based on topology theory [21]. A simple constraint branch chain of a CRR structure was selected, and its transverse and longitudinal kinematic constraint mechanisms formed a 3-DOF parallel mechanism of the $2 \operatorname{SOC}\left\{-C_{i 1}\left\|R_{i 2}\right\| R_{i 3}-\right\},(i=1,2)$ structure. In addition, the rotation between the outer screen frame and the inner screen box formed a 4-DOF

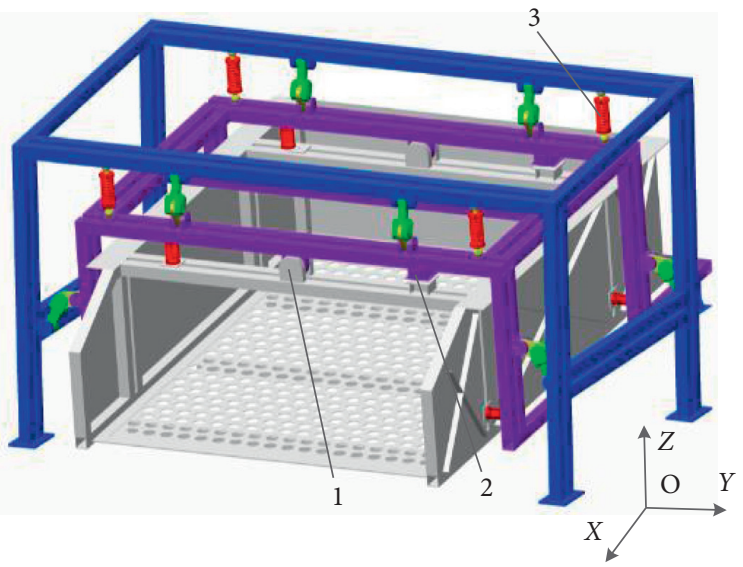

FIgUre 4: Dynamic principle. 1: revolving support device; 2: electromagnetic exciter; 3: suspension spring.

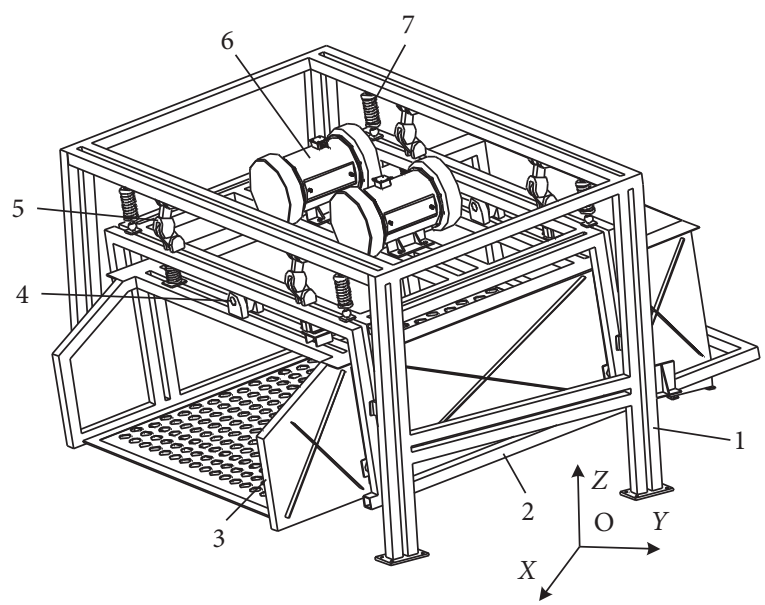

FIGURE 5: Structure of the 4-DOF chaotic vibrating screen. 1: support; 2: outer screen frame; 3: inner screen box; 4: rotary support device; 5: kinematic constraint mechanism; 6: chaotic exciter; 7: bearing spring.

hybrid mechanism of the $4 \operatorname{SOC}\left\{-C_{i 1}\left\|R_{i 2}\right\| R_{i 3}-\right\}+R$, $(i=1,2,3,4)$ structure (the symbol C represents the column pair (equivalent to coaxial $R$ and $P$ ), the symbol $R$ represents the revolute pair, the symbol $P$ represents the moving pair, and symbol $\|$ represents parallel).

The kinematic constraint mechanism of the vibrating screen is schematically presented in Figure 6. The vibrating screen was composed of a lower branched chain of the CRR structure with the symmetrical arrangement of four left and right strips, an upper branched chain of the CRR structure with the symmetrical arrangement of four front and rear strips, and a middle $R$ pair.

The topological structure of left and right constraintbranched chains can be formulated as

$$
\operatorname{SOC}\left\{-\left(P_{i 1} \mid R_{i 2}\right)\left\|R_{i 3}\right\| R_{i 4}-\right\}, \quad i=1,2 .
$$

The topological structure of front and back constraintbranched chains can be formulated as 


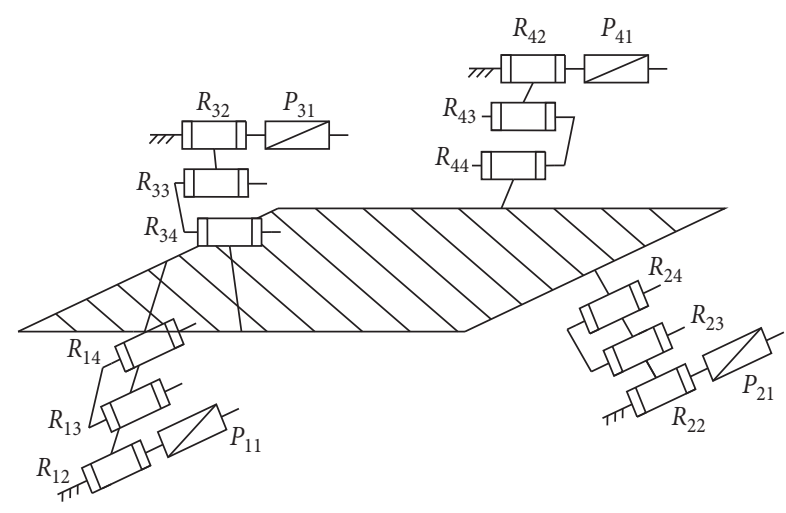

FIGURE 6: Diagram showing the principle of a parallel mechanism.

$$
\operatorname{SOC}\left\{-\left(P_{i 1} \mid R_{i 2}\right)\left\|R_{i 3}\right\| R_{i 4}-\right\}, \quad i=3,4 .
$$

The topological structure of the parallel kinematic constraint mechanism can be expressed as

$$
\operatorname{SOC}\left\{-\left(P_{i 1} \mid R_{i 2}\right)\left\|R_{i 3}\right\| R_{i 4}-\right\}, \quad i=1,2,3,4 .
$$

3.2. Degree-of-Freedom Calculation. Due to the structural symmetry of constraint-branched chains, the model was simplified into a CRR structure constraint-branched chain with two axes being vertical to each other.

(1) Branch topological structure

Constraint-branched chain I: $\operatorname{SOC}\left\{-\left(P_{11} \mid R_{12}\right)\right.$ $\left.\left\|R_{13}\right\| R_{14}-\right\}$

Constraint-branched chain II: $\operatorname{SOC}\left\{-\left(P_{31} \mid R_{32}\right)\right.$ $\left.\left\|R_{33}\right\| R_{34}-\right\}$.

The axes of $R_{12}, R_{13}$, and $R_{14}$ were parallel to each other and the axes of $R_{32}, R_{33}$, and $R_{34}$ were parallel to each other. Moreover, $P_{11} \perp P_{31}$ and $R_{12} \perp R_{32}$, the symbol | represents coaxial, and the symbol $\perp$, |represents vertical axes).

(2) Any point on the outer screen frame (moving platform) of the vibrating screen was selected as the base point $O^{\prime}$ (Figure 7).

(3) The POC sets of the end component of the constraint-branched chains I and II were denoted as $M_{\mathrm{I}}$ and $M_{\mathrm{II}}$, respectively.

$$
\begin{aligned}
M_{b \mathrm{I}} & =\left[\begin{array}{c}
t^{1}\left(\| P_{11}\right) \\
r^{o}
\end{array}\right] \cup\left[\begin{array}{c}
t^{1}\left(\perp R_{12}\right) \\
r^{1}\left(\| R_{12}\right)
\end{array}\right] \cup\left[\begin{array}{c}
t^{1}\left(\perp R_{13}\right) \\
r^{1}\left(\| R_{13}\right)
\end{array}\right] \cup\left[\begin{array}{c}
t^{1}\left(\perp R_{14}\right) \\
r^{1}\left(\| R_{14}\right)
\end{array}\right] \\
& =\left[\begin{array}{c}
t^{1}\left(\| P_{11}\right) \cup t^{1}\left(\perp R_{12}\right) \cup t^{1}\left(\perp R_{13}\right) \cup t^{1}\left(\perp R_{14}\right) \\
r^{1}\left(\| R_{12}\right) \cup r^{1}\left(\| R_{13}\right) \cup r^{1}\left(\| R_{14}\right)
\end{array}\right], \\
M_{b \mathrm{II}} & =\left[\begin{array}{c}
t^{1}\left(\| P_{31}\right) \\
r^{o}
\end{array}\right] \cup\left[\begin{array}{l}
t^{1}\left(\perp R_{32}\right) \\
r^{1}\left(\| R_{32}\right)
\end{array}\right] \cup\left[\begin{array}{l}
t^{1}\left(\perp R_{33}\right) \\
r^{1}\left(\| R_{33}\right)
\end{array}\right] \cup\left[\begin{array}{l}
t^{1}\left(\perp R_{34}\right) \\
r^{1}\left(\| R_{34}\right)
\end{array}\right] \\
& =\left[\begin{array}{c}
t^{1}\left(\| P_{31}\right) \cup t^{1}\left(\perp R_{32}\right) \cup t^{1}\left(\perp R_{33}\right) \cup t^{1}\left(\perp R_{34}\right) \\
r^{1}\left(\| R_{32}\right) \cup r^{1}\left(\| R_{33}\right) \cup r^{1}\left(\| R_{34}\right)
\end{array}\right],
\end{aligned}
$$

where $t^{n}$ represents the fact that the mechanism has $n$ degrees of freedom of movement and $r^{n}$ represents the fact that the mechanism has $n$ degrees of freedom of rotation.

(4) Selection of independent elements of POC sets Both the kinematic chains I and II had four DOF, and thus their POC sets had four independent elements. Each element of the above POC sets can be considered as an independent element; therefore,

$$
\begin{gathered}
M_{b_{1}}=\left[\begin{array}{c}
t^{3} \\
r^{1}\left(\| R_{12}\right)
\end{array}\right], \\
M_{b_{\text {II }}}=\left[\begin{array}{c}
t^{3} \\
r^{1}\left(\| R_{32}\right)
\end{array}\right] .
\end{gathered}
$$

(5) Calculation of DOF of the parallel mechanism According to Figure 7, the number of independent loops is 1 ; hence, the number of independent displacement equation of the loop is 


$$
\begin{aligned}
\zeta L_{\mathrm{I}} & =\operatorname{dim} .\left\{M_{b_{\mathrm{I}}} \cup M_{b_{\mathrm{II}}}\right\}=\operatorname{dim} .\left\{\left[\begin{array}{c}
t^{3} \\
r^{1}\left(\| R_{12}\right)
\end{array}\right] \cup\left[\begin{array}{c}
t^{3} \\
r^{1}\left(\| R_{32}\right)
\end{array}\right]\right\} \\
& =\operatorname{dim} .\left\{\left[\begin{array}{l}
t^{3} \\
r^{2}
\end{array}\right]\right\}=5,
\end{aligned}
$$

where dim represents the dimension of the mechanism. According to the calculation formula of the degree of freedom of the parallel mechanism, we can get

$$
\mathrm{DOF}=\sum_{i=1}^{m} f_{i}-\zeta L_{\mathrm{I}}=(4+4)-5=3,
$$

where $\sum_{i=1}^{m} f_{i}$ is the sum of the degrees of freedom of 8 moving pairs of the simplified parallel mechanism in Figure 7.

(6) The POC equation of the parallel mechanism is

$$
M_{p a}=\left[\begin{array}{c}
t^{3} \\
r^{1}\left(\| R_{12}\right)
\end{array}\right] \cap\left[\begin{array}{c}
t^{3} \\
r^{1}\left(\| R_{32}\right)
\end{array}\right]=\left[\begin{array}{l}
t^{3} \\
r^{0}
\end{array}\right] .
$$

Combined with Figure 4, the additional rotary support device in series is equivalent to a revolute pair with the degree of freedom of 1 , which together with the parallel mechanism constitutes a hybrid mechanism, and its POC equation is

$$
M_{H}=M_{P a} \cup M_{S}=\left[\begin{array}{l}
t^{3} \\
r^{0}
\end{array}\right] \cup\left[\begin{array}{l}
t^{0} \\
r^{1}
\end{array}\right]=\left[\begin{array}{l}
t^{3} \\
r^{1}
\end{array}\right] .
$$

Therefore, the freedom of motion of the inner screen box is $\mathrm{DOF}=3+1=4$.

Hence, it is clear that the outer screen frame of the vibrating screen had three independent translational motions in space, whereas the inner screen box had three independent translational motions and one independent rotation in space.

\section{Dynamic Analysis of the Chaotic Vibrating Screen}

A virtual prototype model of the 4-DOF chaotic vibrating screen was created in Pro/E software and then exported into ADAMS multibody dynamic simulation software for dynamic analysis. The phase trajectory chaotic recognition of the outer screen frame motion was performed in MATLAB.

4.1. Setting of Constraints. Different constraints, such as revolute pair, column pair, fixed pair, and elastic load, were set in ADAMS software, and the driving load was applied on the axis of the rotating motor (Figure 8). A total of 20 revolute pairs, 8 column pairs, 1 fixed pair, and 16 elastic loads with different parameters were created, Among them, the elastic load parameters of the virtual prototype of vibrating screen were set as shown in Table 2 . The driving speed of the chaotic exciter was $1500 \mathrm{rpm}$, the simulation time was $100 \mathrm{~s}$, and the number of simulation steps was 1000 .

4.2. Motion Simulation Analysis. The amplitude, velocity, and acceleration curves against the time of the outer screen frame of the 4-DOF chaotic vibrating screen are displayed in Figure 9.

It is discernible from Figure 9 that under the action of the chaotic exciter, the motor uniformly rotated in one direction, whereas the chaotic exciter had motion outputs in the $X$-, $Y$-, and $Z$-directions, and its motion changed from periodic to aperiodic.

In the $X$-direction (along the length of the screen), the amplitude of the outer screen frame ranged from 12 to $14 \mathrm{~mm}$ and the average amplitude was $8-10 \mathrm{~mm}$. A linear trend was observed in the initial stage of the amplitude curve. After $6 \mathrm{~s}$, the amplitude began to increase. In the range of 55-68 s, the amplitude was large and increased irregularly. Both the velocity time history graph and the acceleration time history graph manifested the characteristics of reciprocating disorder and irregularity.

In the $Y$-direction (along the width of the screen), the amplitude of the outer screen frame ranged from 213 to $240 \mathrm{~mm}$ and the average amplitude ranged from 10 to $12 \mathrm{~mm}$. After $10 \mathrm{~s}$, the amplitude began to increase and the motion characteristic curve became relatively dense. The amplitude of the outer screen frame was large, between 0 and $15 \mathrm{~s}$, and in the range of 65-90 s. Although the amplitude was unstable, the size was controllable. Thus, the amplitude did not increase gradually and damage the vibrating screen. Both the velocity time history graph and the acceleration time history graph manifested the characteristics of reciprocating disorder and irregularity.

In the $Z$-direction (along the vertical direction of the screen), the amplitude of the outer screen frame ranged between -200 and $-50 \mathrm{~mm}$ and the average amplitude was 15 to $20 \mathrm{~mm}$. In the range of $65-85 \mathrm{~s}$, the large amplitude caused a large flee movement due to the light stiffness of the suspension spring. Hence, the motion curve was completely disordered, and no linear relationship was observed. Both the velocity time history graph and the acceleration time history graph manifested the characteristics of reciprocating disorder and irregularity.

The $Y$-direction amplitude was greater than the $X$-direction amplitude. Under the action of the eccentric block, the vibration of the chaotic exciter mainly occurred in the 


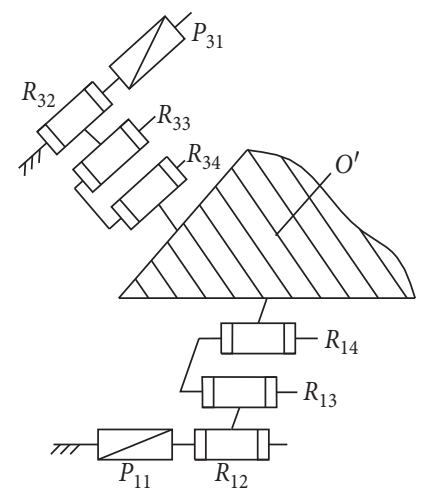

FIgURE 7: Simplified parallel mechanism.

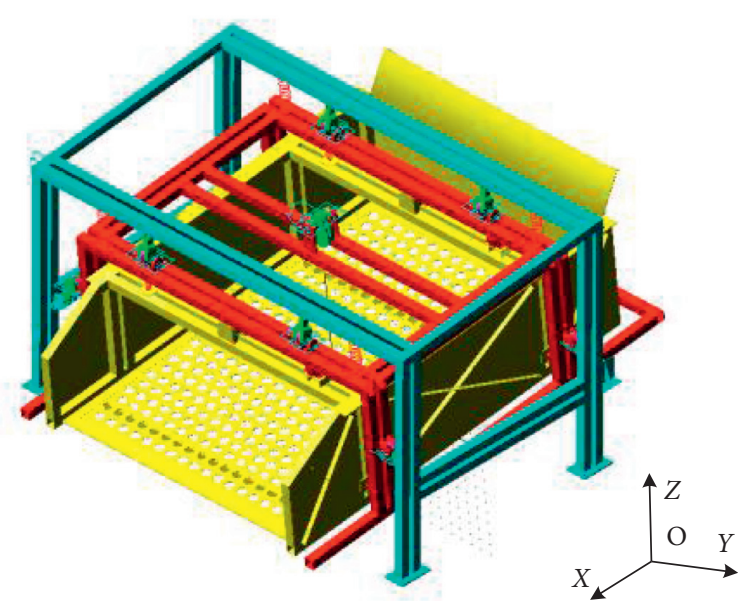

FIGURE 8: Setting of constraints of the virtual prototype of the chaotic vibrating screen.

TABLE 2: Elastic load parameter setting of virtual prototype.

\begin{tabular}{|c|c|c|c|c|}
\hline Parameter connection & Stiffness coefficient & Damping coefficient & Preload & Number \\
\hline Vibrating screen support and outer screen frame are translational & 1000 & No & 0 & 4 \\
\hline The outer screen frame and the inner screen box are translational & 900 & No & 0 & 4 \\
\hline The outer screen frame and the inner screen box are rotational & 1000 & No & 0 & 2 \\
\hline Eccentric block and shell & 1000 & 2.8 (Newton-sec/mm) & 0 & 6 \\
\hline
\end{tabular}

vertical direction to the axis of the rotary motor. The amplitudes of the vibrating screen in the $X$ - and $Y$-directions were changed by adjusting the direction of the chaotic vibration exciter. The maximum amplitude was obtained in the $Z$-direction due to the superposition of the chaos exciter and the suspension spring energy.

Its data curve was locally disordered but overall stable, and it could be used to judge that the motion result was not random vibration and showed reciprocating aperiodic motion. As the translation motions of the outer screen frame and the inner screen box in the $X_{-}^{-}, Y_{-}$, and $Z$-directions were completely consistent, the inner screen box experienced a stable reciprocating aperiodic motion in the three directions.

The aperiodic motion of the outer screen frame and the inner screen box of the vibrating screen was generated by the rotation of the eccentric block driven by the rotary motor in the chaotic exciter. The connecting spring increased the uncertainty of the eccentric block motion and then caused the chaos phenomenon of the outer screen frame and the inner screen box motion in $X$-, $Y$-, and $Z$ - directions.

4.3. Chaos Recognition. The time history chart method with phase trajectories was used to identify whether the chaotic vibrating screen was in a chaotic state [22].

In the postprocessor module of ADAMS software, the simulation data of displacement, velocity, and acceleration in the $X$-, $Y$-, and $Z$-directions of the eccentric block and inner screen box of the chaotic exciter were exported to obtain data text, which was then imported into Excel software and processed in MATLAB to obtain their phase trajectories of displacement, velocity, and acceleration (Figure 10). 


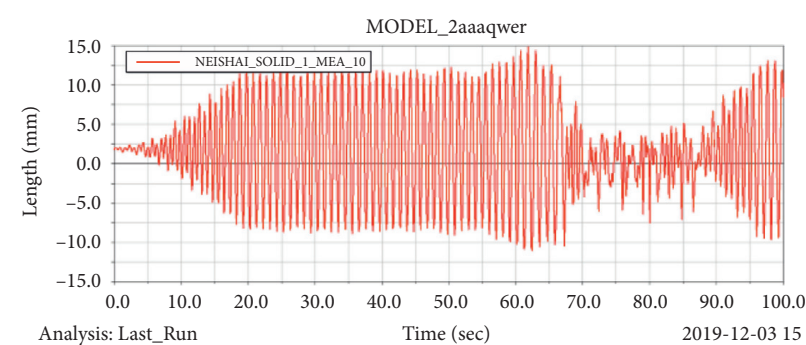

(a)

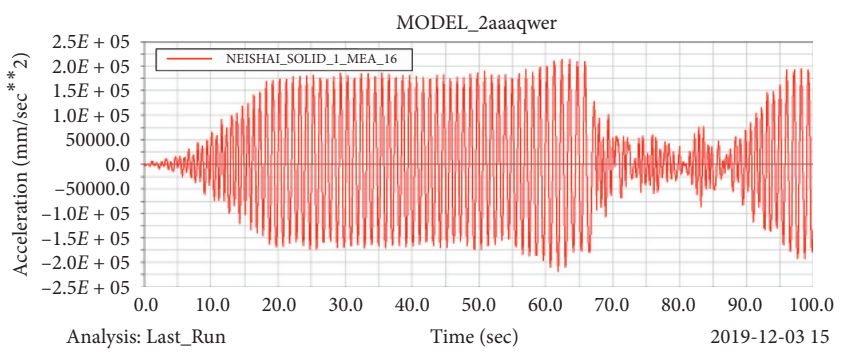

(c)

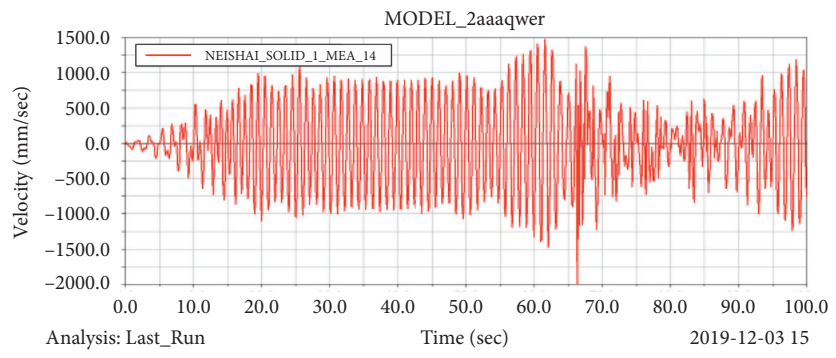

(e)

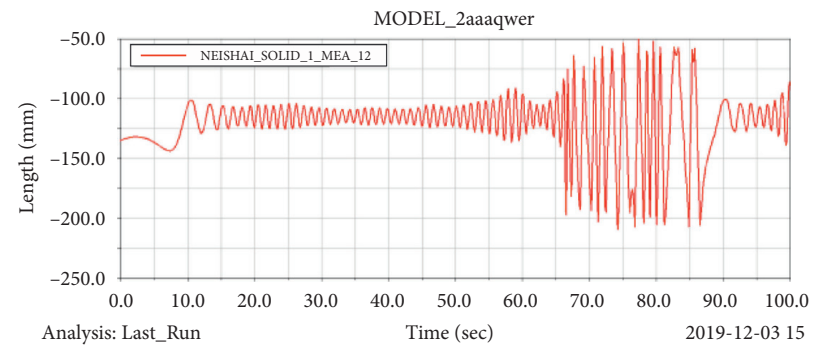

(g)

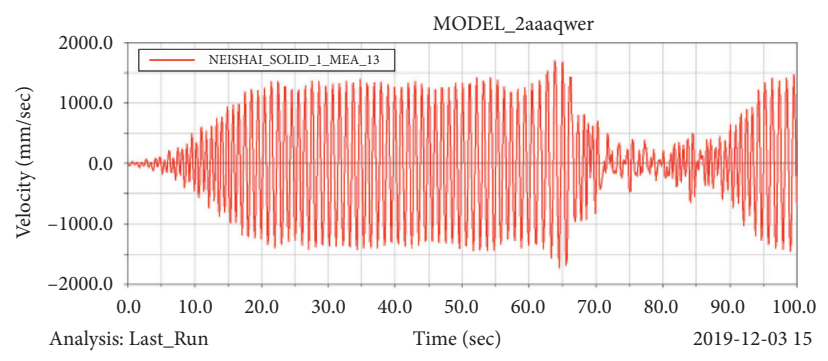

(b)

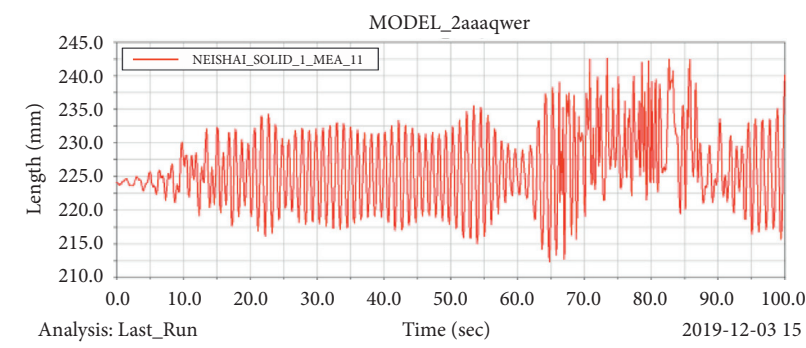

(d)

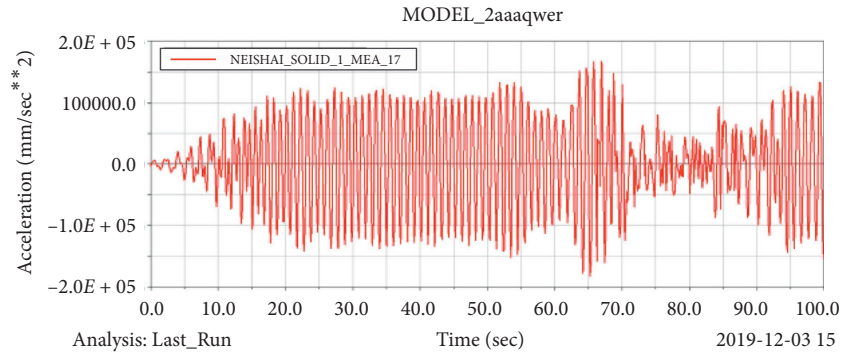

(f)

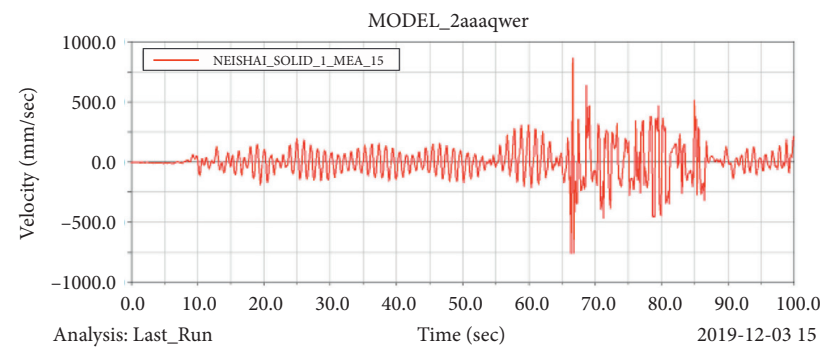

(h)

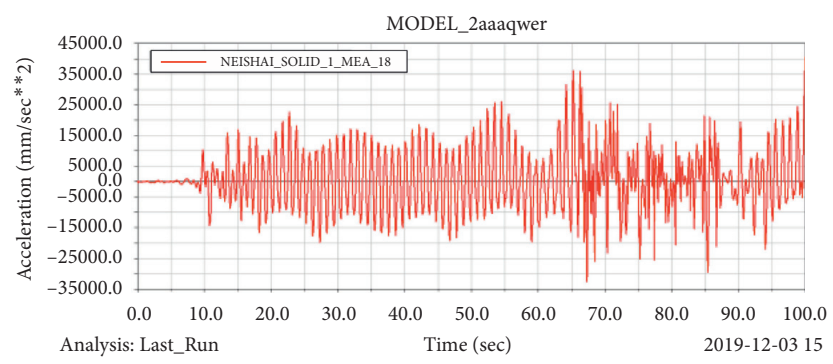

(i)

FIGURE 9: Dynamic simulation results of the outer screen frame of the 4-DOF chaotic vibrating screen. (a) Amplitude curve in the $X$ direction, (b) velocity curve in the $X$-direction, (c) acceleration curve in the $X$-direction, (d) amplitude curve in the $Y$-direction, (e) velocity curve in the $Y$-direction, (f) acceleration curve in the $Y$-direction, (g) amplitude curve in the $Z$-direction, (h) velocity curve in the $Z$ direction, and (i) acceleration curve in the $Z$-direction. 


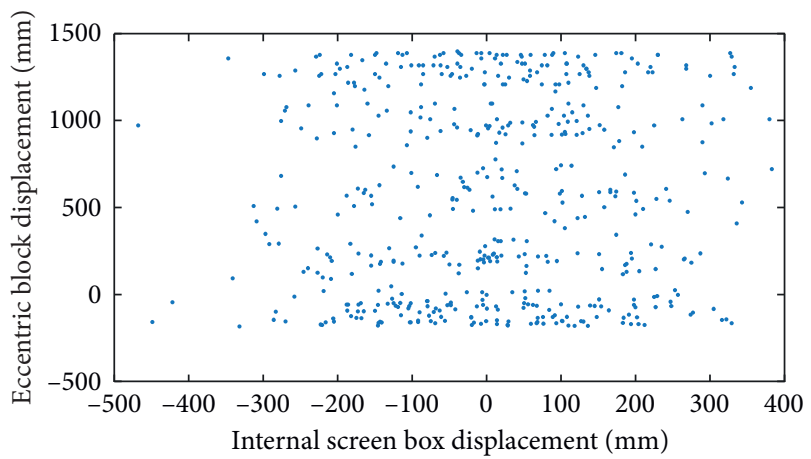

(a)

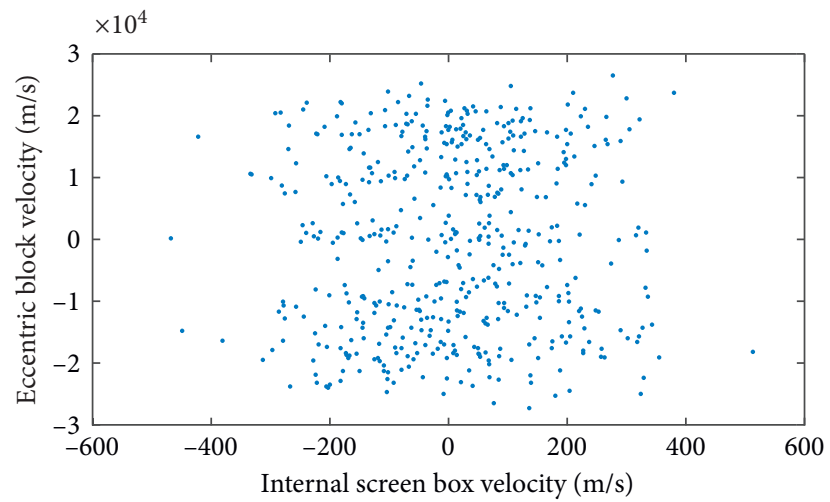

(b)

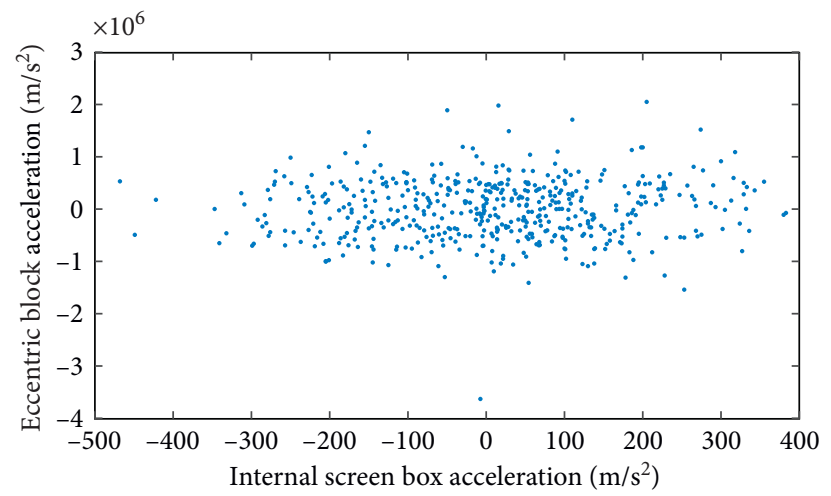

(c)

Figure 10: Phase trajectories of the eccentric block and inner screen box of the chaotic exciter. (a) Displacement phase trajectory, (b) velocity phase trajectory, and (c) acceleration phase trajectory.

The discrete and disordered distribution of the captured data points reveals that the 4-DOF vibration screening system driven by a chaotic exciter was in a chaotic state, which met the design requirements of a chaotic vibrating screen. The designed vibrating screen was a 4 -DOF chaotic vibrating screen, and the translational vibration of the screen surface in the $X$-, $Y$-, and $Z$ directions was chaotic vibration.

\section{Conclusions}

Based on the TRIZ theory, the present paper innovatively designed a 4-DOF (three translational and one rotational movement) chaotic vibrating screen that can be used for sieving and fine sieving of moist materials with high viscosity.

(1) The 4-DOF chaotic vibrating screen was designed by using a nine-screen method and goldfish method. The chaotic exciter was used as the main excitation power source of the vibrating screen. The 3-DOF (three translational movements) parallel mechanism and the 4-DOF (three translational and one rotational movements) hybrid mechanism were used as the kinematic constraint mechanism of the outer screen frame the inner screen box, respectively. The existing vibrating screen screening efficiency is low, and there are easy blocking of screen holes and other problems.

(2) Based on topological theory, the topological structure for the kinematic constraint mechanism of the vibrating screen was established. It was found that the degrees of freedom of the kinematic constraint mechanism of the outer screen frame and inner screen box were 3 and 4 , respectively. The movement freedom of the inner screen box was increased, and the dispersion and screening rate of the screen surface material particles were improved.

(3) The dynamic simulation results of the virtual prototype of a chaotic vibrating screen show that the amplitude, velocity, and acceleration of the inner screen box experienced a large reciprocating aperiodic change. The magnitudes in the $X$ - and $Y$-directions were dependent on the arrangement mode of the chaotic exciter. The action superposition of the suspension spring and the chaos exciter was helpful to improve the excitation effect in the vertical direction. Using ADAMS software to chaos recognition of the inner screen box motion, it was confirmed that the translational vibration of the screen surface of the designed vibrating screen in the $X$-, $Y$-, and $Z$ directions was chaotic vibration, and the designed vibrating screen was chaotic vibration. 
With the widespread application of multidimensional vibration equipment in the material screening industry, discrete element software, finite element software, and multibody dynamics analysis software will be used in the future to conduct a coupled screening test analysis for a variety of materials, especially wet and difficult-to-screen materials. The four-degree-of-freedom chaotic vibrating screen product prototype verifies the screening effect and screening efficiency of the chaotic vibrating screen and promotes the application in developed products.

\section{Data Availability}

The data used to support the findings of this study are included within the article.

\section{Conflicts of Interest}

The authors declare that they have no conflicts of interest.

\section{Acknowledgments}

This research was supported by the National Special Project on Innovative Methods (Funder ID: SQ2018IMC300005) which is greatly acknowledged. The authors thank LetPub (http://www.letpub.com) for its linguistic assistance during the preparation of this manuscript.

\section{References}

[1] J. Gleick, Chaos: A New Science, Social Sciences Literature Press, China, 1991.

[2] A. Algaba, F. Fernández-Sánchez, M. Merino, and A. J. Rodríguez-Luis, "Comments on "Global dynamics of the generalized Lorenz systems having invariant algebraic surfaces"' Physica D: Nonlinear Phenomena, vol. 266, pp. 80-82, 2014.

[3] A. V. Krysko, J. Awrejcewicz, A. A. Zakharova, I. V. Papkova, and V. A. Krysko, "Chaotic vibrations of flexible shallow axially symmetric shells," Nonlinear Dynamics, vol. 91, no. 4, pp. 2271-2291, 2018.

[4] Y.-S. Kim and J.-C. Kim, "Analysis of chaotic vibration of Shilnikov-type in rotor with asymmetric and non-linear stiffness," International Journal of Non-linear Mechanics, vol. 109, pp. 132-139, 2019.

[5] S. Liu, W. xiulei, Ji Wang et al., "Study on chaotic vibration based on double well system," Vibration and Shock, vol. 36, no. 24, pp. 23-29, 2017.

[6] Z. Malanchuk, E. Malanchuk, and V. Korniyenko, "Modeling of vibro screening at fine classification of metallic basalt," Theoretical and Practical Solutions of Mineral Resources Mining, vol. 1, no. 1, pp. 441-443, 2015.

[7] O. A. Makinde, K. Mpofu, and B. Ramatsetse, "Establishment of the best maintenance practices for optimal reconfigurable vibrating screen management using decision techniques," International Journal of Quality \& Reliability Management, vol. 33, no. 8, pp. 1239-1267, 2016.

[8] O. A. Makinde, K. Mpofu, and R. Vrabic, "A bio-inspired approach for the design of a multifunctional robotic endeffector customized for automated maintenance of a reconfigurable vibrating screen," Robotics and Biomimetics, vol. 4, no. 1, pp. 1-29, 2017.
[9] Z. Li, "Chaotic vibration sieve," Mechanism and Machine Theory, vol. 30, no. 4, pp. 613-618, 1995.

[10] Y. Qin, X. Jin, Y. Chen et al., "Research on mechanism of chaotic vibration,” Machine Design, vol. 28, no. 7, pp. 17-20, 2011.

[11] J. Chen, Y. Qin, X. Gong et al., "The response analysis of chaotic vibration screen agencies of speed," Machinery, vol. 42, no. S1, pp. 7-10, 2015.

[12] Z. Wang, N. Wang, Y. Gao et al., "Research on chaotic vibration screen," Mining \& Processing Equipment, vol. 1, no. 3-5, pp. 45-47, 2003.

[13] C. Wang and Y. Long, "Research on chaotic vibration," Journal of China Agricultural University, vol. 6, pp. 23-27, 1997.

[14] C. Wang, T. Zhang, L. Li et al., "Study on screening efficiency of wet raw coal particles in a three degree of freedom hybrid vibrating screen," Journal of Engineering Design, vol. 23, no. 3, pp. 264-270, 2016.

[15] Y. Song, X. Jiang, J. Song et al., "Dynamic analysis of a chaotic vibrating screen," Procedia Earth and Planetary Science, vol. 1, no. 1, pp. 1525-1531, 2009.

[16] G. S. Altshuller, And Suddenly the Inventor Appeared: TRIZ, the Theory of Inventive Problem Solving, Technical Innovation Center, Inc, Worcester, UK, 2nd edition, 1996.

[17] L. Wang, Y. Cui, Z. Zheng et al., "Effect of different motion forms of vibrating screen on particle group screening," Journal of Agricultural Machinery, vol. 50, no. 6, pp. 119-129, 2019.

[18] C. Cheng, J. Fu, Z. Chen et al., "Effect of vibration parameters of vibrating screen of harvester on adhesion characteristics of different humidity effluents," Journal of Agricultural Engineering, vol. 35, no. 8, pp. 29-36, 2019.

[19] Y. Qin, X. Jin, Y. Chen, and J. Chen, "Study on the mechanism of chaotic vibrating screen," Mechanical Design, vol. 28, no. 7, pp. 17-20, 2011.

[20] H. Li, C. Wang, Z. Wang et al., "Finite element analysis of three degree of freedom chaotic vibrating screen," Mining Machinery, vol. 47, no. 6, pp. 41-44, 2019.

[21] T. Yang, Topological Structure Design of Robot Mechanism, Science Press, China, 2012.

[22] Z. Wang, Design and Research of Three Degree of Freedom Chaotic Vibrating Screen, Anhui University, Hefei, China, 2017. 